\title{
On the spatial scaling of soil moisture
}

\author{
Andrew W. Western ${ }^{\mathrm{a}, *}$, Günter Blöschl ${ }^{\mathrm{b}}$ \\ ${ }^{a}$ Centre for Environmental Applied Hydrology, Department of Civil and Environmental Engineering, The University of Melbourne, Australia \\ ${ }^{\mathrm{b}}$ Institut für Hydraulik, Gewässerkunde und Wasserwirtschaft, Technische Universität, Vienna, Austria
}

Received 24 November 1997; accepted 10 September 1998

\begin{abstract}
The spatial scale of soil moisture measurements is often inconsistent with the scale at which soil moisture predictions are needed. Consequently a change of scale (upscaling or downscaling) from the measurements to the predictions or model values is needed. The measurement or model scale can be defined as a scale triplet, consisting of spacing, extent and support. 'Spacing' refers to the distance between samples; 'extent' refers to the overall coverage; and 'support' refers to the area integrated by each sample. The statistical properties that appear in the data, the apparent variance and the apparent correlation length, are as a rule different from their true values because of bias introduced by the measurement scale. In this paper, high-resolution soil moisture data from the 10.5 ha Tarrawarra catchment in south-eastern Australia are analysed to assess this bias quantitatively. For each survey up to 1536 data points in space are used. This allows a change of scale of two orders of magnitude. Apparent variances and apparent correlation lengths are calculated in a resampling analysis. Apparent correlation lengths always increase with increasing spacing, extent or support. The apparent variance increases with increasing extent, decreases with increasing support, and does not change with spacing. All of these sources of bias are a function of the ratio of measurement scale (in terms of spacing, extent and support) and the scale of the natural variability (i.e. the true correlation length or process scale of soil moisture). In a second step this paper examines whether the bias due to spacing, extent and support can be predicted by standard geostatistical techniques of regularisation and variogram analysis. This is done because soil moisture patterns have properties, such as connectivity, that violate the standard assumptions underlying these geostatistical techniques. Therefore, it is necessary to test the robustness of these techniques by application to observed data. The comparison indicates that these techniques are indeed applicable to organised soil moisture fields and that the bias is predicted equally well for organised and random soil moisture patterns. A number of examples are given to demonstrate the implications of these results for hydrologic modelling and sampling design. (C) 1999 Elsevier Science B.V. All rights reserved.
\end{abstract}

Keywords: Soil moisture; Scale; Variogram; Sampling

\section{Introduction}

Soil moisture is a key variable in hydrologic processes at the land surface. It has a major influence on a wide spectrum of hydrological processes including flooding, erosion, solute transport, and landatmosphere interactions (Georgakakos, 1996). Soil

* Corresponding author. moisture is highly variable in space and knowledge of the characteristics of that variability is important for understanding and predicting the above processes at a range of scales.

There are two main sources of data for capturing the spatial variability of soil moisture. These are remote sensing data and field measurements. While remote sensing data potentially give spatial patterns, interpretation of the remotely sensed signal is often 
spacing

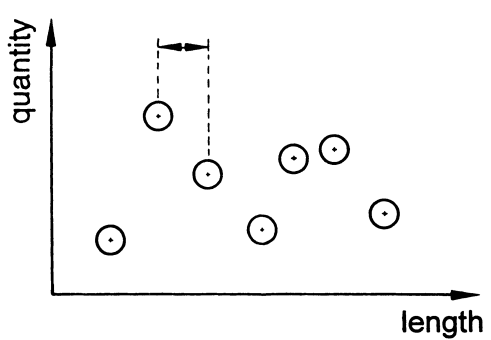

extent

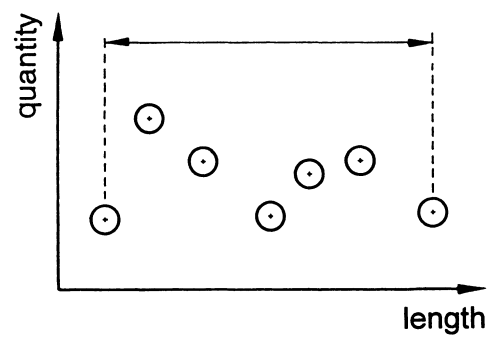

support

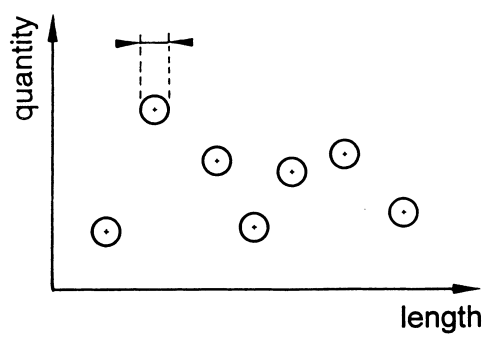

Fig. 1. Definition of the scale triplet (spacing, extent and support). This scale triplet can apply to samples (i.e. measurement scale) or to a model (i.e. modelling scale). After Blöschl and Sivapalan (1995).

difficult. Specifically, there are a number of confounding factors such as vegetation characteristics and soil texture that may affect the remotely sensed signal much more strongly than the actual soil moisture (De Troch et al., 1996). Also, remote sensing signals give some sort of average value over an area (which is termed the footprint) and it is difficult to relate the soil moisture variability at the scale of the footprint to larger scale or smaller scale soil moisture variability (Stewart et al., 1996). A further complication when interpreting soil moisture patterns obtained from microwave images is that the depth of penetration is poorly defined and can vary over the image. This means that the depth over which the soil moisture has been integrated is unknown and may vary. An alternative is to use field data. However, field data are always point samples and, again, it is difficult to relate the point values to areal averages. Also, field data are often collected in small catchments, while soil moisture predictions are needed in large catchments; and the samples are often widely spaced while, ideally, closely spaced samples are needed.

With both remote sensing and field data, these difficulties arise because the scale at which the data are collected is different from the scale at which the predictions are needed. In other words, the difficulty is related to the need for a "change of scale" from the measurements to the predictive model. This change of scale has been discussed by Blöschl (1998) and Beckie (1996), within the conceptual framework of scaling. Upscaling refers to increasing the scale and downscaling refers to decreasing the scale. Blöschl (1998) noted that the variability apparent in the data will be different from the true natural variability and that the difference will be a function of the scale of the measurements. Similarly, the variability apparent in the parameters or state variables of a model will be different from the true natural variability (and from the variability in the data), and this difference will be a function of the scale of the model.

Blöschl and Sivapalan (1995) suggested that both the measurement scale and the modelling scale consist of a scale triplet consisting of spacing, extent, and support (Fig. 1). 'Spacing' refers to the distance between samples or model elements; 'extent' refers to the overall coverage; and 'support' refers to the integration volume or area. All three components of the scale triplet are needed to uniquely specify the space dimensions of a measurement or a model. For example, for a transect of TDR (time domain reflectometry) soil moisture samples in a research catchment, the scale triplet may have typical values of, say, $10 \mathrm{~m}$ spacing (between the samples), $200 \mathrm{~m}$ extent (i.e. the length of the transect), and $10 \mathrm{~cm}$ support (the diameter of the region of influence of the TDR measurement). Similarly, for a finite difference model of spatial hydrologic processes in the same catchment, the scale triplet may have typical values of, say $50 \mathrm{~m}$ spacing (between the model nodes), $1000 \mathrm{~m}$ extent (i.e. the length of the model domain), and $50 \mathrm{~m}$ support (the size of the model elements or cells).

Blöschl and Sivapalan (1995) and Blöschl (1998) noted that the effect of spacing, extent, and support can be thought of as a filter and should always be viewed as relative to the scale of the natural variability. The scale of the natural variability is also termed the 'process scale' and relates to whether the natural 


\section{UPSCALING}
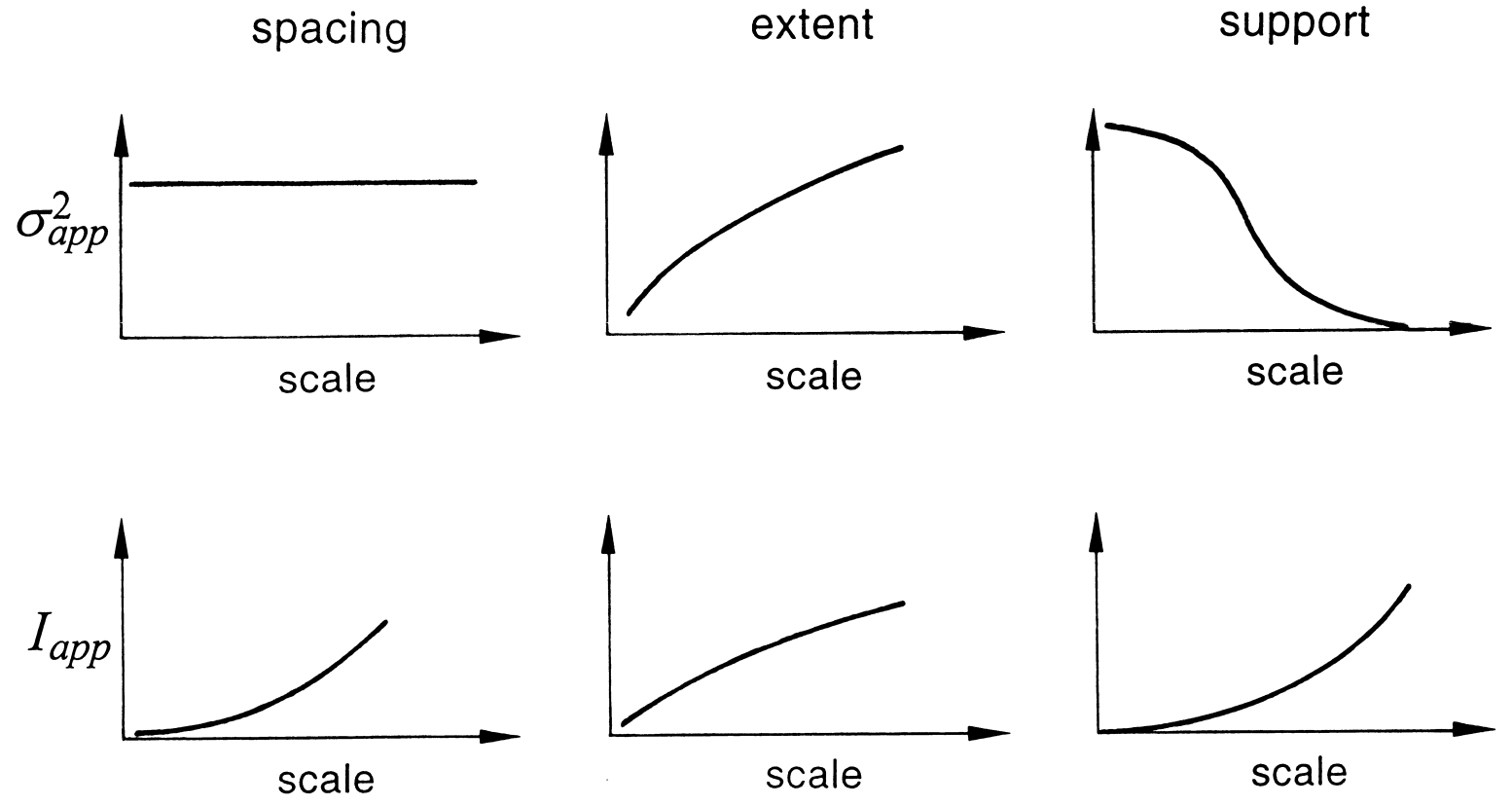

Fig. 2. Effect of the measurement scale (or modelling scale) on the apparent variance and the apparent integral scale. Schematic after Blöschl (1998).

variability is small-scale variability or large-scale variability. More technically, the correlation length or the integral scale of a natural process (Journel and Huijbregts, 1978) can quantify the scale of the natural variability (the process scale). The correlation length can be derived from the variogram or the spatial covariance function of the data.

The variogram characterises spatial variance as a function of the separation (lag) of the data points. The main structural parameters of the variogram are the sill and correlation length. The sill is the level at which the variogram flattens out. If a sill exists, the process is stationary and the sill can be thought of as the variance of two distantly separated points. The correlation length is a measure of the spatial continuity of the variable of interest. For an exponential variogram, the correlation length relates to the average distance of correlation. The spatial correlation scale is sometimes characterised by the range instead of the correlation length. The range is the maximum distance of which spatial correlations are present. While the correlation length and the range contain very similar information, the numerical value of the range is three times the correlation length for an exponential variogram.

As mentioned previously, the spatial variability apparent in the data will be different from the true spatial variability. Here, we are interested in the bias in the statistical properties of the true spatial variability, estimated from the measured data. First, the apparent variance in the data will, as a rule, be biased as compared to the true variance, and this bias is a function of the ratio of measurement scale and process scale. If the support of the measurement scale is large as compared with the process scale (the true correlation length), most of the variability will be averaged out and the apparent variance will be smaller than the true variance. This is consistent with the 


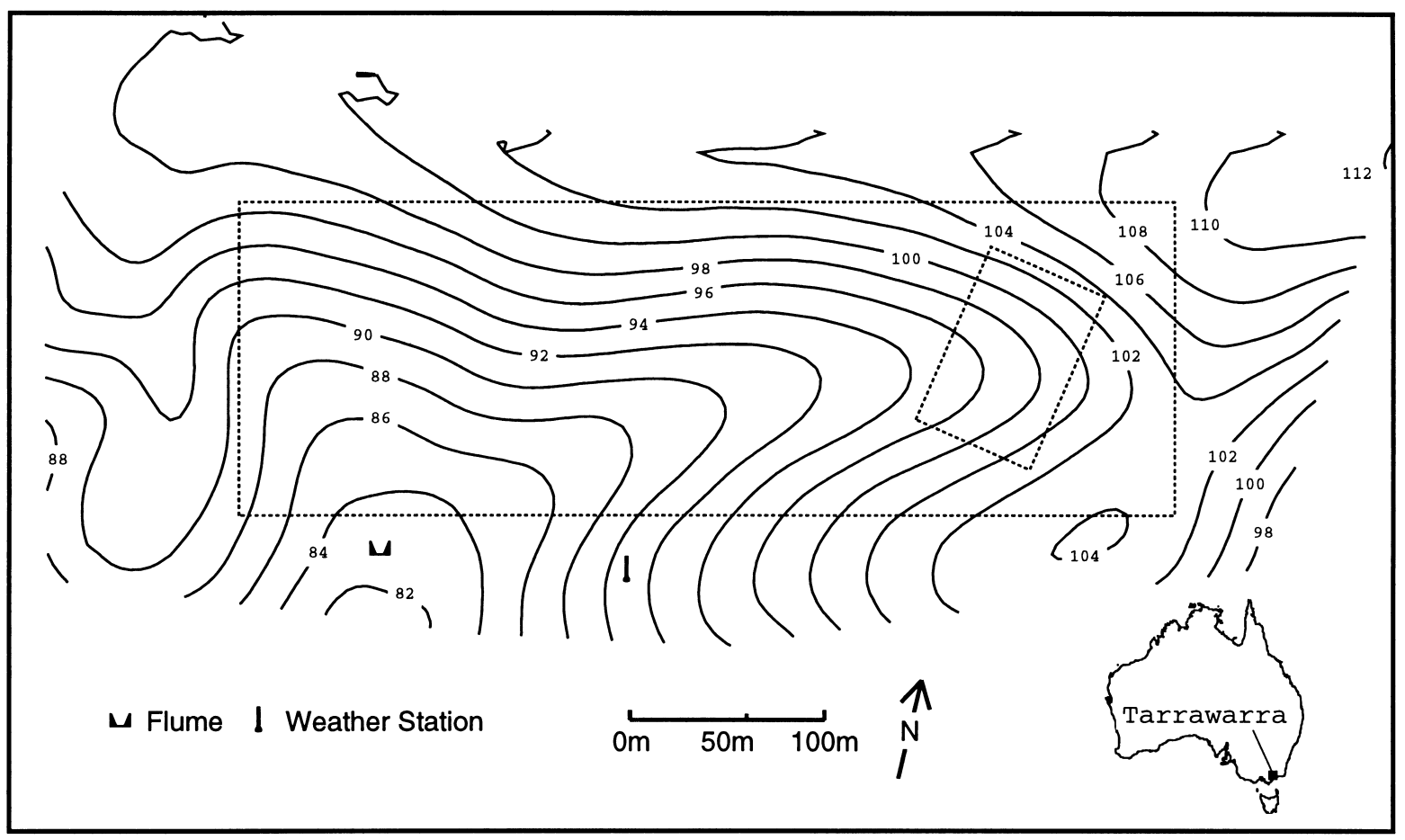

Fig. 3. Location map of the Tarrawarra catchment near Melbourne, Australia. The two sampling plots are outlined.

general observation that aggregation always removes variance. However, if the extent is small as compared to the process scale, the large-scale variance is not sampled and the apparent variance will be smaller than the true variance. The bias associated with extent and support is depicted schematically in Fig. 2. Second, the apparent correlation length (or apparent integral scale) in the data will, as a rule, be biased as compared with the true correlation length. Again, this bias is a function of the ratio of measurement scale and process scale. It is clear that large-scale measurements can only sample large-scale variability and small-scale measurements can only sample smallscale variability. As a consequence of this, large measurement scales (in terms of spacing, extent and support), compared to the process scale, will generally lead to apparent correlation lengths that are larger than the true correlation lengths, and small measurement scales will cause an underestimation of the correlation lengths (Fig. 2). The effect of the modelling scale (in terms of spacing, extent and support) will be similar to that of the measurement scale.
While, conceptually, it is straightforward to assess bias related to measurement scale, it may be difficult to estimate it quantitatively. One approach is to use a geostatistical framework (Journel and Huijbregts, 1978; Isaaks and Srivastava, 1989; Gelhar, 1993; Vanmarcke, 1983;). In geostatistics, the spatial variability is represented by the variogram, which is the lag dependent variance of the natural process. Based on the variogram, there are a number of geostatistical techniques available that allow quantitative estimates of each bias mentioned earlier. For example, for analysing the effect of support, regularisation techniques are given in the literature. All of these techniques hinge on the assumption of the variable under consideration being a spatially correlated random variable. However, for the case of soil moisture this is not necessarily a valid assumption. There is substantial evidence from measurements in the literature that soil moisture is indeed spatially organised (Dunne et al., 1975; Rodríguez-Iturbe et al., 1995; Georgakakos, 1996; Schmugge and Jackson, 1996; Western et al., 1998a). For example, soil moisture is often 
Table 1

Summary of the four soil moisture patterns from Tarrawarra used in this paper (full data set)

\begin{tabular}{lllllllll}
\hline Survey & Date & Grid & No. of points used & Area $\left(\mathrm{m}^{2}\right)$ & Mean $(\% \mathrm{~V} / \mathrm{V})$ & $\sigma_{\text {true }}^{2}(\% \mathrm{~V} / \mathrm{V})^{2}$ & $\lambda_{\text {true }}(\mathrm{m})$ & Random/organised \\
\hline S1 & 27 Sep. 95 & $10 \times 20 \mathrm{~m}$ & $24 \times 16=384$ & 76800 & 38.6 & 18.3 & 28 & $\mathrm{O}$ \\
$\mathrm{S} 3$ & 23 Feb. 96 & $10 \times 20 \mathrm{~m}$ & $24 \times 16=384$ & 76800 & 21.1 & 4.7 & 14 & $\mathrm{R}$ \\
$\mathrm{S} 7$ & 2 May 96 & $2 \times 2 @ 10 \times 20 \mathrm{~m}$ & $48 \times 32=1536$ & 76800 & 42.2 & 16.4 & 22 & $\mathrm{O}$ \\
Fine & 25 Oct. 96 & $2 \times 2 \mathrm{~m}$ & $32 \times 48=1536$ & 6144 & 40.9 & 21.7 & 12 & $\mathrm{O}$ \\
\hline
\end{tabular}

topographically organised with connected bands of high soil moisture in the depression zones of a catchment and near the streams. Soil moisture may also be organised as a consequence of landuse patterns, vegetation patterns, soil patterns, geology and other controls.

This paper has two aims. The first is to examine how the apparent spatial statistical properties of soil moisture (variance and correlation length) change with the measurement scale (in terms of spacing, extent and support). The second is to examine whether standard geostatistical techniques of regularisation and variogram analysis are applicable to organised soil moisture patterns. The main feature of this paper is that we use soil moisture data collected in the field with a very high spatial resolution. Real spatial patterns are used because they often have characteristics that do not conform to the assumptions underlying the standard geostatistical approach, yet standard geostatistics are often applied. Of greatest significance here is the existence of connectivity and nonstationarity. These characteristics introduce uncertainty about the applicability of standard geostatistical tools for scaling spatial fields such as soil moisture. Stationary random fields, which are often assumed in geostatistics, do not have these characteristics. Using real data allows assessment of the robustness of the predictions of standard geostatistical techniques to the presence of spatial organisation (connectivity). We are not aware of any paper that examines the effect of connectivity on scaling. The data we use also allows consideration of a range of scales of two orders of magnitude in the analysis.

\section{Field description and data set}

The data used to examine the spatial scaling of soil moisture come from the 10.5 ha Tarrawarra catchment. Tarrawarra is an undulating catchment located on the outskirts of Melbourne, Australia (Fig. 3). It has a temperate climate and the average soil moisture is high during winter and low during summer. Very detailed measurements of the spatial patterns of soil moisture have been made in this catchment. In this paper, data from four soil moisture surveys (Table 1) are used. Two of these surveys (S1 and S3) consist of measurements on a 10 by $20 \mathrm{~m}$ sampling grid. In one survey (S7), measurements were made on the corners of a square with side-length $2 \mathrm{~m}$ and the squares were centred on a 10 by $20 \mathrm{~m}$ grid. While the original data cover the entire catchment only those data in a rectangle with side-lengths $480 \times 160 \mathrm{~m}$ are used in this paper to facilitate the resampling analysis (Fig. 3). The remaining survey (fine) consists of measurements on a 2 by $2 \mathrm{~m}$ sampling grid in a $64 \times 96 \mathrm{~m}$ rectangle located over the upstream portion of the eastern drainage line (Fig. 3). Fig. 4 shows the soil moisture patterns of the four surveys.

All measurements were made using time domain reflectometry equipment mounted on an all terrain vehicle. Each measurement represents a point measurement of the moisture in the top $30 \mathrm{~cm}$ of the soil profile. The soils at Tarrawarra have a $20-35 \mathrm{~cm}$ deep A horizon, which is believed to be the hydrologically active zone from the perspective of lateral subsurface flow. Perched water tables form in the A horizon during winter months and the soil profile dries to a depth of approximately $1 \mathrm{~m}$ during summer. The Tarrawarra catchment is used for cattle grazing and has pasture vegetation throughout the catchment. The catchment and data collection methods are described in detail by Western et al. (1997) and Western and Grayson (1998).

The soil moisture surveys used in this paper cover the range of soil moisture conditions typically observed in this landscape. Table 1 gives the summary 


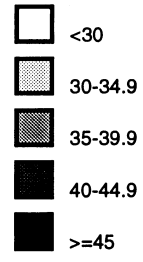

$=45$

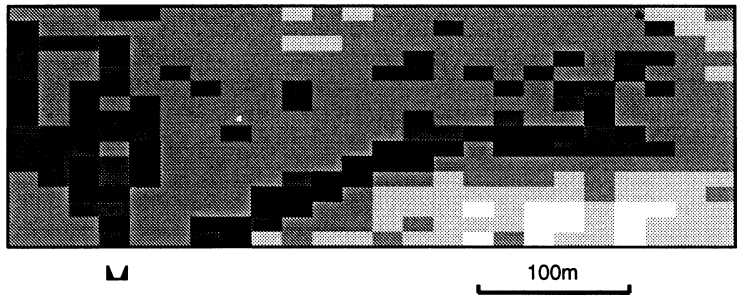

(S1, 27 September 1995)
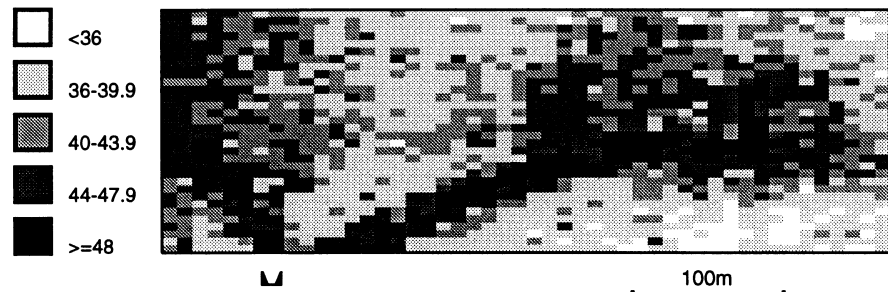

(S7, 2 May 1996)
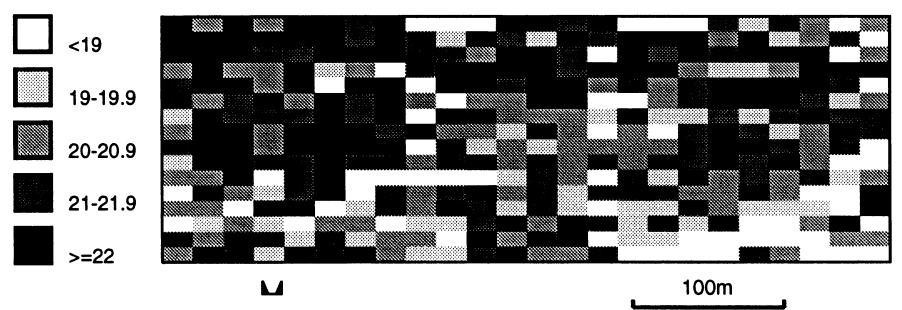

(S3, 23 February 1996)

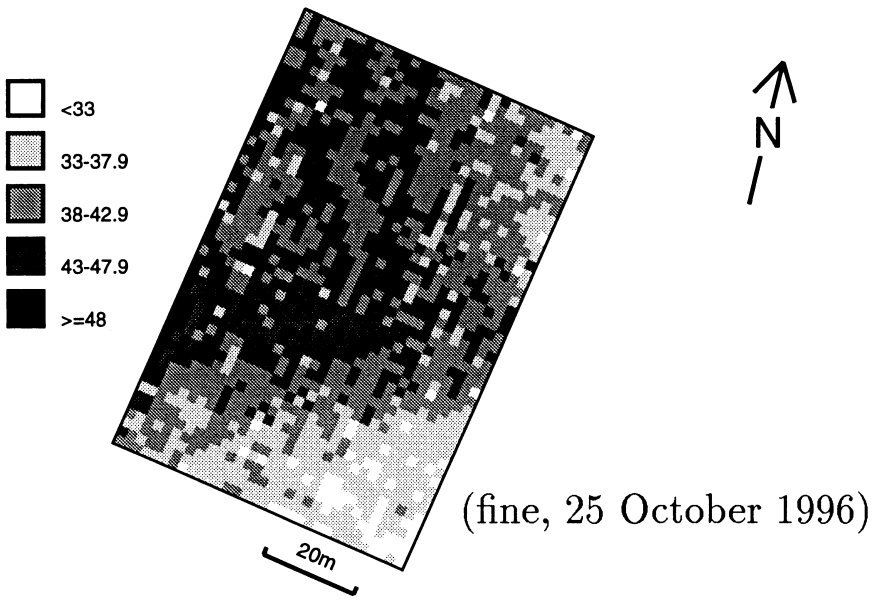

Fig. 4. Measured volumetric soil moisture patterns at Tarrawarra on 27 Sep. 1995 (S1), 23 Feb. 1996 (S3), 2 May 1996 (S7) and 25 Oct. 1996 (fine), expressed as a percentage. Note that S1, S7 and "fine" exhibit spatial organisation while S3 is random. 


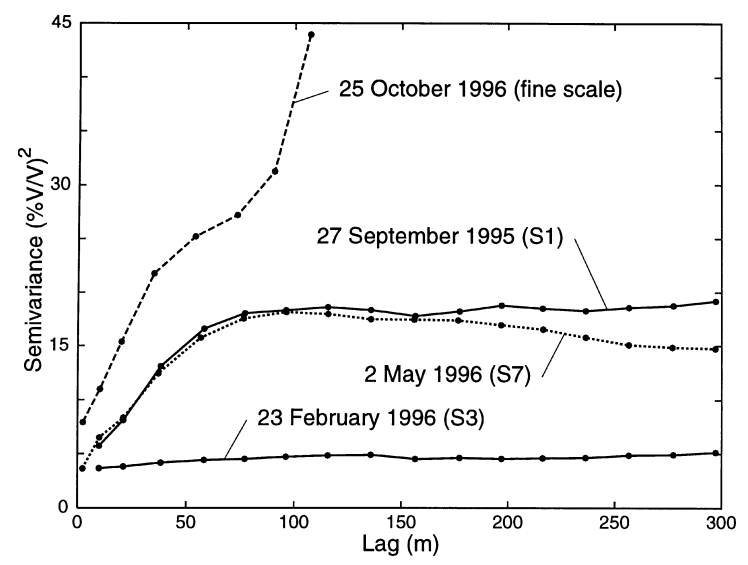

Fig. 5. True variograms of the four soil moisture patterns of Fig. 4.

statistics for the soil moisture data used here. The coefficient of variation varies between 9 and $11 \%$. The soil moisture measurement error variance is 2.9 $(\% \mathrm{~V} / \mathrm{V})^{2}$. Measurement error accounts for less than $20 \%$ of the total variance in all cases, except for the dry case (S3). In the dry case (S3), the error variance is significant $(62 \%)$; however, this case is of least importance to our conclusions since it is the case that most closely meets the underlying assumptions of the geostatistical analyses applied (It is stationary and it doesn't exhibit connectivity). It is included for completeness. Western et al. (1998a) discuss the spatial organisation of soil moisture at Tarrawarra. The measured soil moisture patterns do exhibit spatial organisation the degree of which varies seasonally. During wet periods there is a high degree of organisation. This organisation consists of connected band of high soil moisture in the drainage lines and is due to lateral redistribution of water by both surface and subsurface flow paths. During dry periods there is only a little spatial organisation and the spatial variation appears to be mainly random. Three of the surveys used here are representative of organised conditions (S1, S7, fine) and one is representative of random conditions (S3).

\section{Method of analysis}

The analysis in this paper consists of three main steps. The first step is an analysis of the full data set, which gives the "true", variogram. The second step is a resampling analysis in which the (empirical) variance and integral scale are estimated. The third step consists of estimating the (theoretical) variance and integral scale directly from the "true" variogram.

In all three steps, an exponential variogram without a nugget is used. Western et al. (1998b) found that the soil moisture data at Tarrawarra are fitted well by exponential variograms with a nugget; however, here the nuggets are neglected. The reason for doing this is that, in practice, there are often insufficient data for inferring accurate nugget values and the nugget is assumed to be zero. Also, this allows a more robust estimation of the integral scale. A second assumption is that of local stationarity, i.e. a sill exists for all variograms irrespective of the scale considered. The sill is assumed to be equal to the variance of the data at that scale. Again, this is a pragmatic assumption often made in practice, and it allows a more robust estimation of the integral scale.

\subsection{Analysis of the full data set}

From the four data sets of soil moisture (Fig. 4), empirical variograms were calculated. These are shown in Fig. 5. It is clear from Fig. 5 that the surveys on 27 September 1995 and 2 May 1996 (S1 and S7) have variograms that are close to exponential and the nuggets are small as compared to the sill. These are the surveys where the soil moisture is topographically organised (Table 1). The 23 February 1996 survey (S3) has a relatively large nugget as compared with the sill and the range is not well defined. This is the survey where the spatial distribution of soil moisture is random (Table 1). The 25 October 1996 survey (fine) is clearly not stationary. This is because only a part of the catchment has been sampled and the maximum lag is not much larger than the range one would expect when comparing this survey to S1 and S7. Also, the variance of the 25 October 1996 survey (fine) is large. This is caused by the location of the sampled domain in an area of topographic convergence. In this area there is significantly more topographic variability, compared with the average topographic variability in the entire catchment. This causes the large value of the soil moisture variance.

Exponential variograms (Eq. A1) were fitted to the empirical variograms in Fig. 5. This fitting was achieved by minimising the weighted root mean 


\section{UPSCALING}

\section{SCALE}

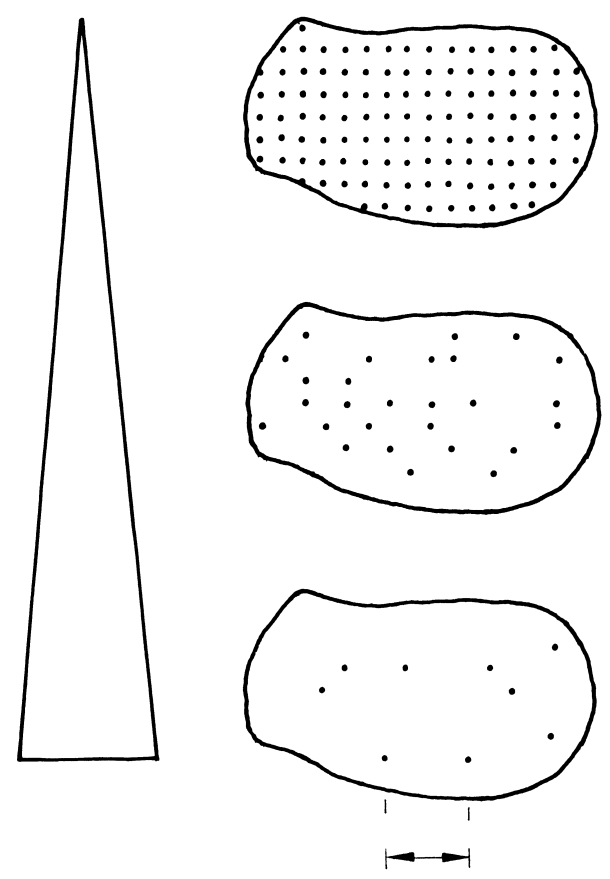

extent
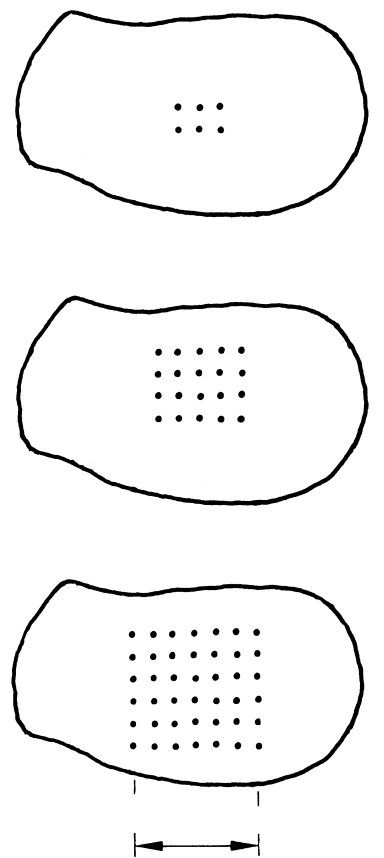

support
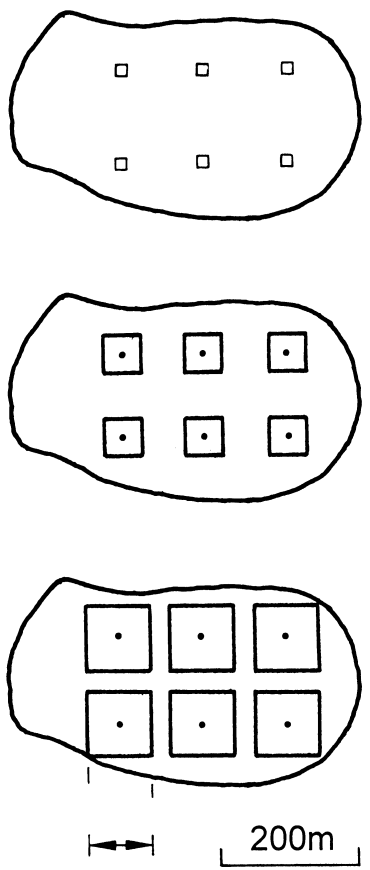

Fig. 6. Schematic of the resampling analysis.

square error over all the lag classes. The error for each class was weighted by the number of pairs in that class. However, the weighting did not significantly affect the results of the fitting. The correlation length $\lambda_{\text {true }}$ was the only parameter optimised. The sill was set to the sample variance and the nugget was assumed to be zero. The fitted correlation lengths $\lambda_{\text {true }}$ are given in Table 1. These variograms are referred to as "true" variograms in the remainder of this paper. They are termed "true" because they are based on the full data set, rather than on part of the data set, which is the case for the variograms estimated in the resampling analysis.

\subsection{Resampling analysis}

The aim of the resampling analysis is to emulate hypothetical sampling scenarios (or model scenarios) for which only a fraction of the full data set is available (Fig. 6). The hypothetical sampling scenarios differ in terms of the scale of the samples. In the framework used here, scale consists of a scale triplet - spacing, extent, and support. Hence there are three cases, each with a range of different measurement scales, considered in the resampling analysis. For each subsample the variance and correlation length were calculated. The variance, $\sigma_{a p p}^{2}$, is simply calculated as the sample variance. The correlation length, $\lambda_{\text {app }}$, is calculated by fitting an exponential variogram, $\gamma_{a p p}$, (with zero nugget and sill equal to the variance) to the empirical variogram of the subsample using the same fitting technique as for the "true" variogram.

$\gamma_{a p p}=\sigma_{a p p}^{2} \cdot\left(1-\exp \left(\frac{-h}{\lambda_{a p p}}\right)\right)$ 
Table 2

Resampling analysis for the cases of spacing, extent and support

\begin{tabular}{|c|c|c|c|c|c|c|}
\hline & $\begin{array}{l}\text { Minimum-spacing } \\
\text { No. of points }\end{array}$ & Spacing (m) & No. of realisations & $\begin{array}{l}\text { Maximum-spacing } \\
\text { No. of points }\end{array}$ & Spacing (m) & No. of realisations \\
\hline S1 & 384 & 14.1 & 1 & 6 & 113 & 64 \\
\hline S3 & 384 & 14.1 & 1 & 6 & 113 & 64 \\
\hline S7 & 1536 & 7.1 & 1 & 6 & 113 & 256 \\
\hline \multirow[t]{2}{*}{ fine } & 1536 & 2.0 & 1 & 6 & 32 & 256 \\
\hline & Minimum-extent & Extent (m) & No of realisations & Maximum-extent & Extent $(\mathrm{m})$ & No of realisations \\
\hline & & & & & & \\
\hline S1 & 4 & 28.2 & 96 & 384 & 277 & 1 \\
\hline S3 & 4 & 28.2 & 96 & 384 & 277 & 1 \\
\hline S7 & 4 & 4.0 & 384 & 1536 & 277 & 1 \\
\hline \multirow[t]{3}{*}{ fine } & 4 & 4.0 & 384 & 1536 & 78.4 & 1 \\
\hline & Minimum-support & & & Maximum-support & & \\
\hline & No. of points & Support (m) & No. of points aggregated & No. of points & Support (m) & No. of points aggregated \\
\hline S1 & 384 & Small & 1 & 6 & 113 & 64 \\
\hline S3 & 384 & Small & 1 & 6 & 113 & 64 \\
\hline S7 & 1536 & Small & 1 & 6 & 113 & 256 \\
\hline fine & 1536 & Small & 1 & 6 & 32 & 256 \\
\hline
\end{tabular}

Variance and correlation length estimated for the subsamples are termed "apparent" variance and "apparent" correlation length, respectively. They are termed " apparent" because they are the values one would typically obtain in practical field studies where the data points are available at a single scale only. The apparent variance and correlation length are, as a rule, different from the true variance and correlation length and this reflects the bias introduced by the measurement or the model. The apparent integral scale, $I_{a p p}$, is equal to the apparent correlation length as estimated from the apparent variogram since the variogram is exponential with no nugget.

$I_{\text {app }}=\lambda_{\text {app }}$

The results of the resampling analysis for the spacing, extent, and support cases are summarised in Table 2. In each case, a range of scales is considered by varying of one component of the scale triplet (Table 2).

In the case of spacing, $n$ points are drawn randomly from the true patterns. The variogram is estimated from the $n$ point samples. Random sampling without replacement then continues until all $N$ point measurements for that survey have been sampled. The variogram is estimated each time. This results in $n_{\text {real }, s p}=$
$N / n$ realisations. The correlation length at a give scale is estimated as the arithmetic average of the $n_{\text {realsp }}$ realisations for that scale. Similarly, the variance at a given scale is calculated as the arithmetic average of the variances of the $n_{\text {realsp }}$ realisations. For survey S1, for example, the scenarios start from $n=\mathrm{N}=384$ points. This means that all the points of the array of $24 \times 16$ points are sampled and one variogram is estimated. For the next scenario, half the number of points $(n=192)$ is used, with $n_{\text {real,sp }}=2$ variograms being estimated and their parameters averaged, and so forth until a minimum value of $n=6$ points. The scale in terms of the spacing, $a_{S p a c}$, is defined as the average spacing of the points:

$a_{\text {Spac }}=\sqrt{A / n}$

where $A$ is the area of the domain as shown in Table 1.

In the case of extent, the first scenario uses all of the points. In the next scenario, the domain is subdivided into three contiguous regions and the data from each region are considered to be one realisation. This means that, for survey S1 for example, each of the three realisations consists of an array of $8 \times 16$ points. From each of these three realisations, the variogram is 

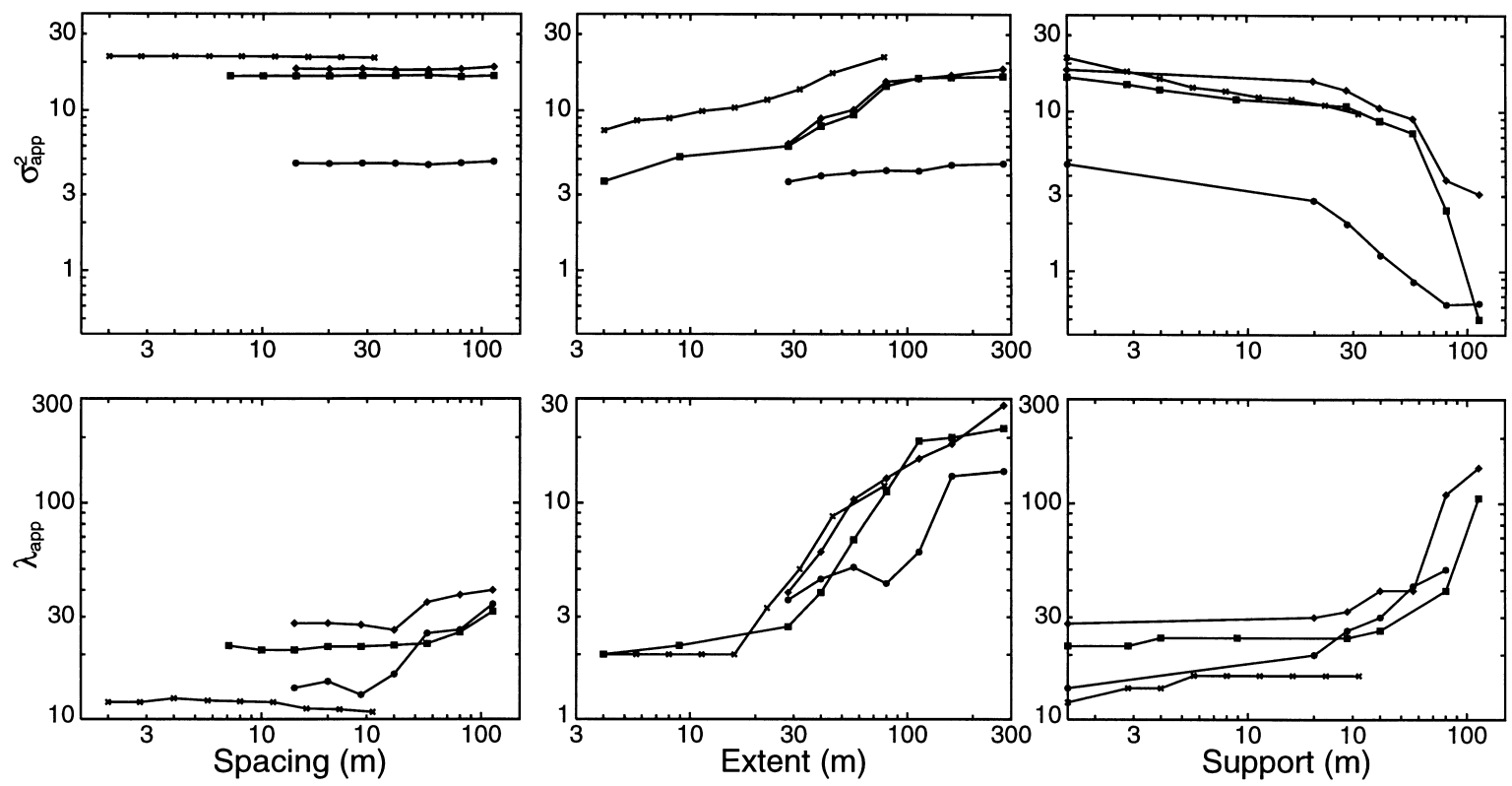

Fig. 7. Results of the resampling analysis: apparent variance and the apparent correlation length (or integral scale) as a function of spacing, extent and support. Diamonds: 27. Sep. 1995; disks: 23. Feb. 1996; squares: 2 May 1996; crosses: 25 Oct. 1996 (fine).

estimated and the correlation length at that scale is estimated as the arithmetic average of the correlation lengths of the three realisations. In the next scenario, the domain is subdivided into six contiguous regions and so forth until a maximum value of 96 regions (for $\mathrm{S} 1)$. In the last scenario each region only contains 4 points (Table 2). The scale in terms of the extent, $a_{E x t}$, is defined as the square root of the area of the region, $A_{\text {region: }}$ :

$a_{\text {Ext }}=\sqrt{A_{\text {region }}}$

Since the total area $A$ is fully tessellated into regions, the number of regions (or realisations) is $n_{\text {real }, e x}=A / A_{\text {region }}$ for each scenario. The variance at a given scale is calculated analogously to the correlation length as the arithmetic average of the variances of the $n_{\text {real }, e x}$ realisations.

In the case of support, the first scenario uses each point individually. In the next scenario, two adjacent points are aggregated (by arithmetic averaging) into one mean value. This means that, for survey $\mathrm{S} 1$ for example, the variogram is estimated from 192 aggregated values that are on a $20 \times 20 \mathrm{~m}$ grid. In the next scenario, four adjacent points are aggregated and so forth until a minimum value of 6 aggregated values. In the case of support there is no averaging of correlation lengths or variances as in all scenarios there is only a single realisation. The scale in terms of the support, $a_{\text {Supp }}$, is defined as the square root of the area over which the samples are aggregated, $A_{\text {aggreg }}$ :

$a_{\text {Supp }}=\sqrt{A_{\text {aggreg }}}$

Arithmetic averaging of point samples into aggregated values is consistent with the conservation of mass of soil moisture.

\subsection{Estimating the (theoretical) variance and integral scale directly from the "true" variogram}

The rationale for calculating the variance and integral scale directly from the "true" variogram is to examine whether standard geostatistical techniques of regularisation and variogram analysis are applicable to the case of soil moisture patterns. This is done because soil moisture patterns exhibit spatial organisation that violates the spatially random behaviour assumed by the standard geostatistical techniques. The methods and equations used to calculate the 

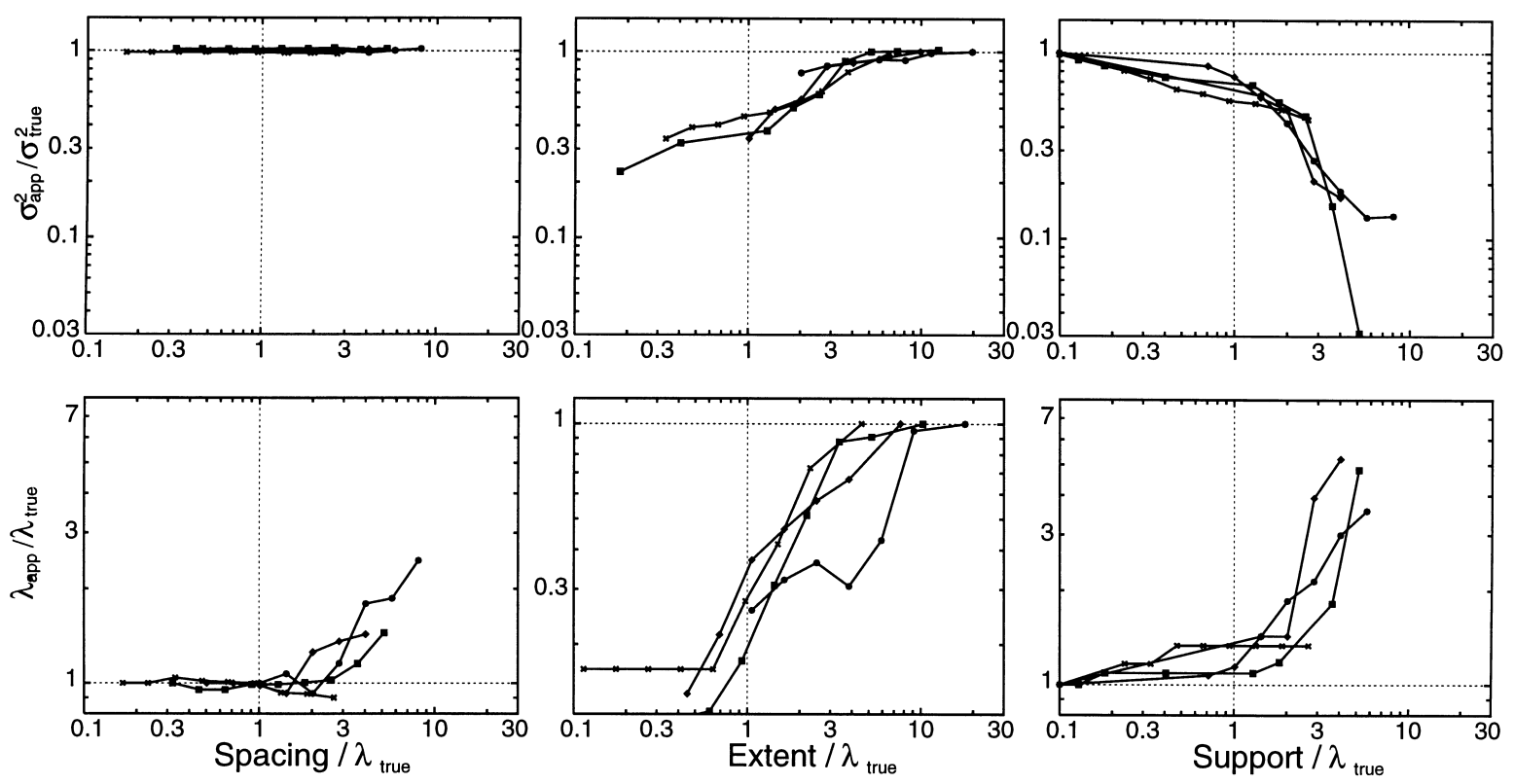

Fig. 8. As for Fig. 7; however, all scales have been normalised by the true correlation length, $\lambda_{\text {true }}$ and the apparent variances have been normalised by the true variances, $\sigma_{\text {true }}^{2}$.

apparent variance, $\sigma_{a p p}^{2}$, and the apparent integral scale, $I_{\text {app }}$, for each of the cases of the scale triplet support, extent and spacing are summarised in Appen$\operatorname{dix}$ A.

In the case of support, standard regularisation techniques are used that calculate the variogram of an averaged process using the variogram of the point process and a filter function. The filter is represented by a square of side length $a_{\text {Supp }}$, which is the support. The square is the area over which the aggregation takes place. Different shapes of this area do not significantly affect the results of the regularisation (Rodríguez-Iturbe and Mejía, 1974). In the case of spacing, the apparent variogram is approximated by the true variogram for lags larger than the spacing $a_{\text {Spac }}$ and by a linear increase from the origin for shorter lags. This assumption is made because when estimating the empirical variogram there are only a small number of pairs of points for lags smaller than the spacing (Russo and Jury, 1987) and a straight line is the simplest approximation. However, this is a very simple assumption, particularly for small lags, and it may be worth examining the shape of the apparent variogram more closely for the spacing case in future studies. Discussions of related work are given in
Russo and Jury (1987) and Gelhar (1993). In the case of extent, the apparent variogram is based on the true variogram for small lags [for $\gamma(h) \leq \sigma_{a p p}^{2}$ ], and is constant and equal to $\sigma_{a p p}^{2}$ for large lags. For the extent case, nugget effects can be important for small extents. Therefore, as an exception, the apparent variances and integral scales are also derived for exponential variograms with non-zero nuggets.

In the cases of spacing and extent, the apparent variances, $\sigma_{a p p}^{2}$, can be given analytically, and the apparent integral scales, $I_{a p p}$, are derived from the apparent variograms, $\gamma_{a p p}$, by analytical integration:

$I_{a p p}=\int_{0}^{\infty} 1-\frac{\gamma_{a p p}(x)}{\sigma_{a p p}^{2}} \mathrm{~d} x$

In the case of support, numerical integration is required (Appendix A).

\section{Results}

Fig. 7 shows the results of the resampling analysis. Measurement scales in terms of spacing, extent and support have different effects on the apparent variance. Spacing does not affect the apparent 

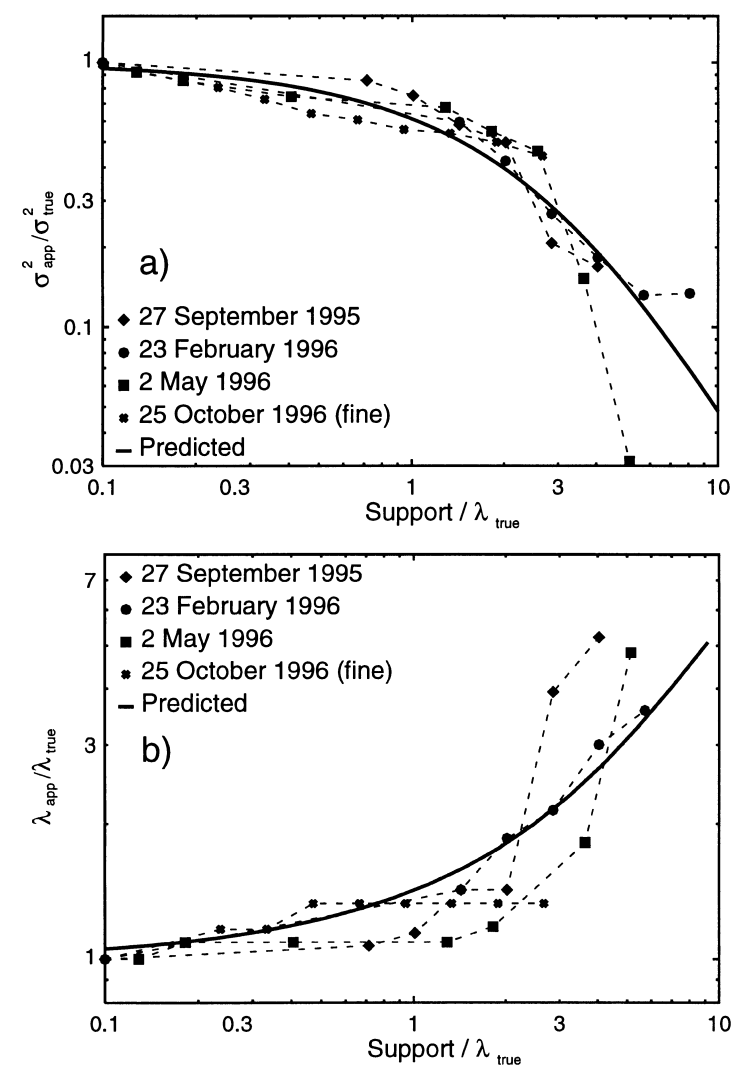

Fig. 9. Effect of support: (a) Comparison of apparent variances from the resampling analysis (markers) with apparent variances predicted by Eq. A7 (line). (b) Comparison of apparent correlation lengths from the resampling analysis (markers) with apparent integral scales predicted by Eq. A9 (line).

variance. Increasing the extent causes an increase in the apparent variance while increasing the support causes a decrease in the apparent variance. On the other hand increasing the scale in terms of spacing, extent and support always increases the apparent correlation length. These tendencies are similar for all the four surveys analysed (Diamonds - 27 September 1995; disks - 23 February 1996; squares - 2 May 1996; crosses — 25 October 1996, fine). However, there is substantial scatter about these general trends. Part of the scatter may be related to the true variance and the true correlation lengths, which are different for the four surveys. Therefore, the information in Fig. 7 has been replotted in a non-dimensional form. The non-dimensional relationships are shown in Fig. 8. The apparent variance has been normalised by the true variance, and the apparent correlation length has been normalised by the true correlation length. Similarly, the measurement scale (in terms of spacing, extent and support) has been normalised by the true correlation length. It is clear that a significant portion of the scatter is removed by the normalisation, particularly for the variance case and to a lesser degree for the correlation length case. The difference between the variance and correlation length cases is related to the relative robustness of the two statistical measures. Variance is a much more robust statistical quantity than the correlation length and hence is estimated more accurately in the resampling analysis. The overall trend of the effect of measurement scale on the apparent variance and the apparent correlation length is similar to that in Fig. 7 but now, the effects can be discussed more quantitatively.

Spacing has no effect on the apparent variance but does affect the apparent correlation length. Once the spacing exceeds about twice the true correlation length, the apparent correlation length will be biased and the bias may be up to a factor of three, for the conditions considered here. Extent may have a significant effect on both statistical quantities. As long as the extent is larger than about 5 times the true correlation length, the bias in both variance and correlation length is small. However, if the extent is small, the apparent variance may be as small as $20 \%$ of the true value and the apparent correlation length may be as small as $10 \%$ of the true value, for the conditions considered here. Similarly, support may have a significant effect on both statistical quantities. As long as the support is smaller than about $20 \%$ of the true correlation length, the bias in both variance and correlation length is small. However, if the support is large, the apparent variance may be as small as $3 \%$ of the true value and the apparent correlation length may be as large as four times the true value, for the conditions considered here. It is clear that all of these effects are significant, with the effect of extent on the correlation length and the effect of support on the variance being the most important ones.

The following analysis examines whether the bias due to sampling scale can be predicted by standard geostatistical techniques of regularisation and variogram analysis. These techniques assume stationary random fields and our aim is to test the robustness 

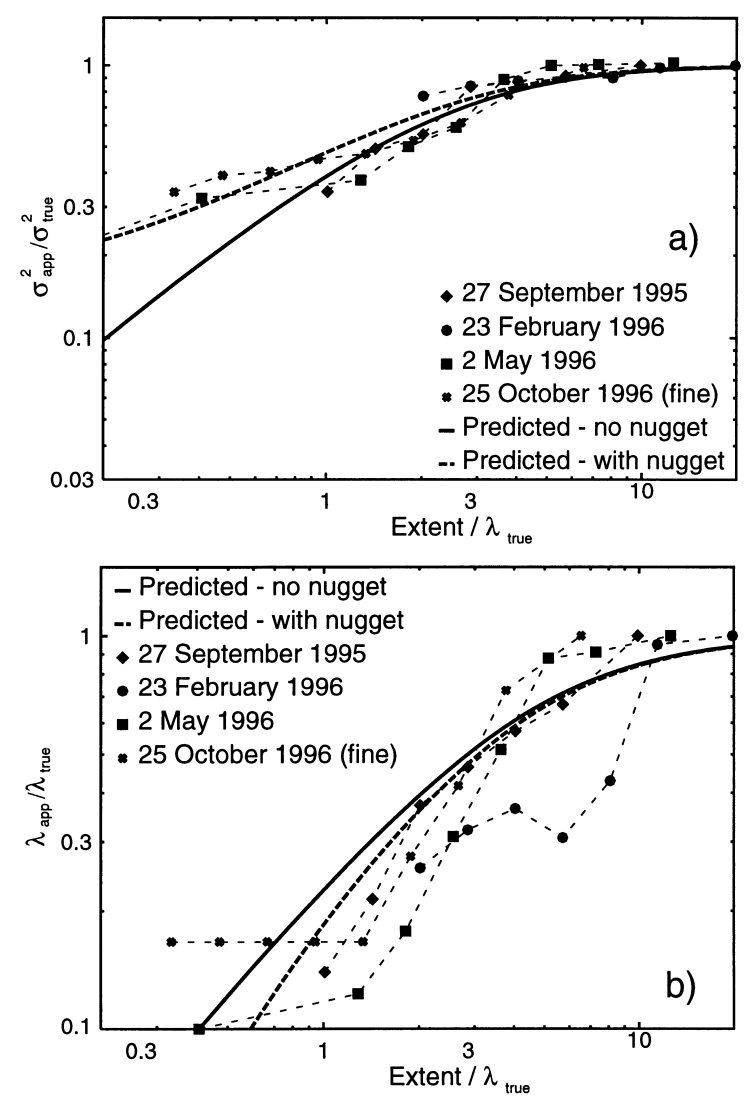

Fig. 10. Effect of extent: (a) Comparison of apparent variances from the resampling analysis (markers) with apparent variances predicted by Eqs. A10 and A19 (lines). (b) Comparison of apparent correlation lengths from the resampling analysis (markers) with apparent integral scales predicted by Eqs. A13 and A20 (lines). Solid lines are for zero nuggets and dashed lines are for a normalised nugget of $\sigma_{\text {nug }}^{2} / \sigma_{\text {true }}^{2}=0.14$.

of these techniques to violations of these assumptions. Here, these assumptions are violated by the presence of connectivity and nonstationarity. The analysis involves a comparison of the apparent variance $\sigma_{a p p}^{2}$ from the resampling analysis with the apparent variance $\sigma_{a p p}^{2}$ predicted by the geostatistical techniques in appendix A. The analysis also involves a comparison of the apparent correlation length $\lambda_{\text {app }}$ from the resampling analysis (which is equal to the apparent integral scale as an exponential variogram with zero nugget is assumed) with the apparent integral scale $I_{a p p}$ predicted by the geostatistical techniques in Appendix A.

Fig. 9(a) and (b) shows the comparison of the resampling analysis with predicted bias for the case of support. Fig. 9(a) indicates that the variances can be predicted very well by standard regularisation techniques. There is essentially no difference between the random case (23 Feb.) and the other cases in terms of the goodness of fit of the predictive relationship. The predictions of the apparent correlation lengths [Fig. 9(a)]are also reasonably close to those estimated from the resampling analysis. Part of the difference between estimated and predicted correlation lengths in Fig. 9(b) is due to the assumption of local stationarity in the resampling analysis. For example, correlation lengths for 25 Oct. (fine) should increase once the support exceeds the true correlation length. Failure to do so is related to an underestimation of the true correlation length of this survey (Table 1) associated with the limited extent of the actual data sampled and the assumption of stationarity of this survey (Fig. 5). The random survey (Feb. 23) is slightly better predicted than the organised surveys.

Fig. 10(a) and (b) shows the comparison of the resampling analysis with predicted bias for the case of extent. The predicted bias is shown for both zero nugget and a normalised nugget of $\sigma_{\text {nug }}^{2} / \sigma_{\text {true }}^{2}=0.14$. This is the normalised nugget one obtains when fitting an exponential variogram (with nugget) to the true data set of the survey of 2 May 96 (S7) (Western et al., 1998b). The normalised nuggets for the other surveys are 0.12 (27 Sept. 95, S1), 0.64 (23 Feb. 96, S3), and 0.29 (25 Oct.; fine). Fig. 10(a) indicates that variances can be predicted quite well by standard variogram analysis techniques. There is essentially no difference between the random case (23 Feb.) and the other cases in terms of the goodness of fit of the predictive relationship. The main difference between the surveys appears to be related to the normalised nugget rather than to the effect of spatial organisation. Indeed, the normalised apparent variance in Fig. 10(a) should never drop below the normalised nugget which can be quite large as in the case of the $23 \mathrm{Feb}$. survey $\left(\sigma_{\text {nug }}^{2} / \sigma_{\text {true }}^{2}=0.63\right)$. While bias is significant for extents smaller than about 5 times the true correlation length, nugget only influences bias for extents smaller than the true correlation length, for most of the surveys considered here (S1, S7, fine). The predictions of the apparent integral scale [Fig. 10(a)] are not as good as those of the apparent variance. The integral scales for the random 


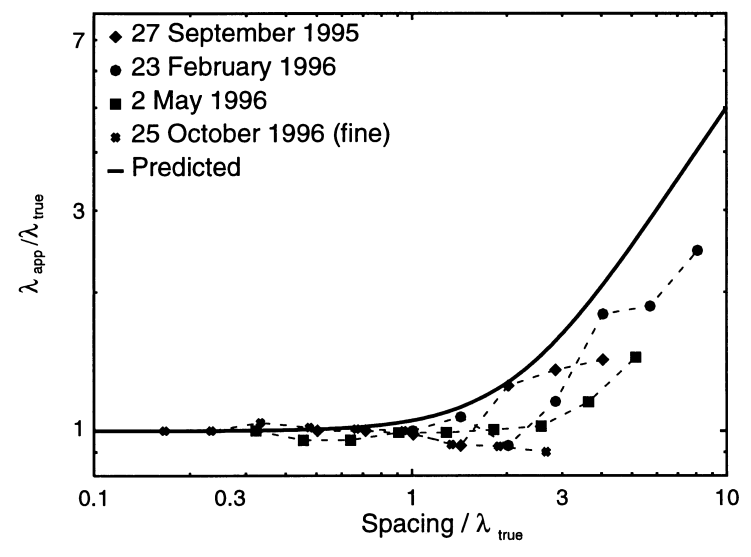

Fig. 11. Effect of spacing: Comparison of apparent correlation lengths from the resampling analysis (markers) with apparent integral scales predicted by Eq. A16 (line).

case (23 Feb. 96; S3) are not well predicted at all and this may be related to poorly defined variograms for that date. Part of the poor fit may also be related to the way the results of the resampling analysis have been normalised in Fig. 10(b). Specifically, the extent has been normalised by the "true" correlation length found by fitting Eq. A1 to the full data set. Using the correlation length for a non zero nugget variogram (Eq. A17) for normalisation would have shifted the curve for 23 Feb. 96 (S3) to the left. However, this has not been done for consistency with the other cases. This sort of resampling analysis inevitably involves some degree of arbitrariness. It is also interesting to note that nugget has only a minor effect on the apparent correlation lengths and that there is no consistent evidence that the presence of spatial organisation in the soil moisture patterns affects the predictive power of the standard variogram analysis techniques used here.

Fig. 11 shows the comparison of the resampling analysis with the predicted bias in the correlation length for the case of spacing. The apparent correlation lengths are always overestimated by the standard regularisation techniques used here. This may be caused by the approximation to the apparent variogram chosen here (Eq. A15) where the apparent variogram was assumed to be linear for short lags. In the resampling analysis, the fitting of the variogram [Eq. (1)] to the empirical variogram extrapolated more accurately to shorter lags than a simple assumption of linearity would predict. As a result of this, there is less bias in the resampling analysis than in the theoretical relationships given in the appendix. However, it should be noted that these results depend substantially on the method used for estimating the correlation length in the resampling analysis. Here theoretical (exponential) variograms were fitted to the empirical variograms. There are a number of other techniques available in the literature such as non-parametric estimation of the correlation length (or the integral scale). Non-parametric methods may have substantially larger bias than those predicted in Fig. 11 (Russo and Jury, 1987). It is also clear form Fig. 11 that there is no significant difference between the predictive power of the variogram analysis techniques for organised and random surveys.

\section{Discussion}

The resampling analysis of the soil moisture data at Tarrawarra indicates that bias in the variance and the correlation length does exist as a consequence of the measurement scale. The general shapes of curves representing bias are as suggested by Blöschl (1998). For the ideal case of very small spacings, very large extents and very small supports, the apparent variance and the apparent correlation length are close to their true values. However, as the spacing increases, the extent decreases or the support increases, bias is introduced.

The exception is the effect of spacing on the apparent variance. The apparent variance does not change with spacing. This can be interpreted in the frequency domain. Large spacings mean that the natural variability is resolved only at low frequencies and high frequencies are not resolved. However, this does not imply that the total spectral variance decreases as the high frequencies are "folded back" to the lower frequencies. This effect is termed "aliasing" in sampling theory (Vanmarcke, 1983; Jenkins and Watts, 1968). This is also consistent with sampling effects as discussed for the time domain in hydrology (Matalas, 1967). Spacing does have a significant effect on apparent correlation lengths and, again, this can be interpreted in the frequency domain. Large spacings cause an overestimation of the correlation length because the sampling only resolves the 
low frequencies (large scales). This is discussed in detail for the groundwater case in Gelhar (1993) and for the soils case in Russo and Jury (1987). Russo and Jury (1987) performed a resampling analysis similar to the one performed here; however, they used synthetically generated random fields (which don't exhibit connectivity), rather than real soil moisture patterns as used in this paper. They used two different methods for estimating the integral scale. The first method was to fit theoretical variograms to empirical variograms in a way very similar to the one used in this paper. Using this method, they found a bias in the integral scale such that $I_{\text {app,Spac }} / \lambda_{\text {true }}=1.1$ and 1.7 for normalised spacings of $a_{\text {Spac }} / \lambda_{\text {true }}=3.1$ and 4.4, respectively (their Table 1). This is very close to the values found in the resampling analysis of this paper (Fig. 11). The second method was to find the integral scale by numerical integration of the empirical variogram, i.e. a non-parametric method. The second method gave a much larger bias in the integral scale. For normalised spacings of $a_{S p a c} / \lambda_{\text {true }}=3.1$ and 4.4 , $I_{\text {app }, \text { Spac }} / \lambda_{\text {true }}=2.7$ and 3.4 , respectively. This is larger than the values predicted by Eq. A16, as shown in Fig. 11. Clearly, parametric methods are more robust than non-parametric methods, provided the shape of the variograms is known.

The effect of the decreasing extent was to decrease apparent variance and to decrease apparent correlation length. This can also be interpreted in the frequency domain. Small extents mean that the natural variability is sampled at high frequencies only and the low frequencies are not sampled. As a consequence of this, the total spectral variance is lower and the integral scale is biased towards high frequencies (small scales). The same interpretation can be made from the variogram. The important assumption in the extent case is that of stationarity. Local stationarity and how this is related to global variability has long puzzled hydrologists (Klemeš, 1974). There is a general observation that natural variability increases with the scale (extent) of the observation (Feder, 1988) and it is not straightforward to reconcile this with the assumption of local stationarity. One interpretation has been given by Gelhar (1986); Fig. 8, where he suggested that the global variogram may consist of nested locally stationary variograms. However, the interpretation made in this paper is that local stationarity is useful as a working hypothesis, even though it may not necessarily be consistent with global behaviour. Another aspect of the effect of extent is the quantitative effect on the apparent correlation length. For the case of hydraulic conductivity in aquifers, data given in Gelhar (1993); Fig. 6.5, indicate that the apparent correlation length is about $10 \%$ of the extent, and similar results were found in Blöschl (1998) for the case of snow cover patterns in an Alpine catchment in Austria. This is actually quite consistent with the results for soil moisture in this paper [Fig. 10(b)], at least for extents smaller than $10 \times \lambda_{\text {true }}$. However, it is not clear whether this consistency is an indication of some universal behaviour of hydrological processes or an artefact of the sampling and the statistical analyses. It should also be noted that the flattening out of the relationships in Fig. 10(a) and (b) for extents larger than $10 \times \lambda_{\text {true }}$ may be related to the limited extent of the data used in this paper. It is likely that, as the scale (extent) of the domain increases, both the soil moisture variability and the scale of that variability (i.e. the integral scale) would increase. This is because at scales that are beyond the maximum scale examined in this study (10 ha) one may expect that other sources of variability come in. One potentially very important source is different landuse types. Landuse is relatively uniform at Tarrawarra. Other sources of variability are differences in vegetation, soil type and geology. 1997a).

The effect of increasing support was to decrease apparent variance and to increase correlation length. Clearly, part of the variability is smoothed out when real averages rather than point values, are considered. It is interesting to note that the predictive relationships (regularisation) used here both for the variance and the correlation length were much closer to the results of the resampling analysis than in the spacing and the extent cases. This is partly due to the predictive relationships used in the spacing and the extent cases being approximations. Part may also be due to the averaging, where an increase in support increases the robustness of the estimates in the resampling analysis. A number of studies have examined the change of apparent variance with changing support for soil moisture estimates derived from remotely sensed data. For example, Rodríguez-Iturbe et al. (1995) analysed soil moisture data derived from ESTAR measurements of the subhumid Little 
Washita watershed in south-west Oklahoma, USA during the Washita '92 Experiment. They examined the variance reduction observed as $200 \times 200 \mathrm{~m}$ pixels were aggregated up to 1 by $1 \mathrm{~km}$ and concluded that soil moisture exhibited power law behaviour with an exponent $\alpha$ between -0.21 and -0.28 in the relationship:

$\sigma_{\text {app }, \text { Supp }}^{2}=A_{\text {aggreg }}^{\alpha}$

This is equivalent to an exponent of between -0.42 and -0.56 in a relationship between apparent variance and support, $a_{\text {Supp }}$. This is equivalent to a straight line in Fig. 9(a) with a slope of between -0.42 and -0.56 . Although the relationships found here are not straight lines in Fig. 9(a), the section of the plot from a support of about $0.3 \times \lambda_{\text {true }}$ to about $3 \times \lambda_{\text {true }}$, can be approximated by a straight line with a slope in the range of -0.42 to -0.56 . However, it should be noted that Rodríguez-Iturbe et al. (1995) examined only a very limited range of supports. While the range of supports considered here is much larger (two orders of magnitude), it is important to note that the shapes of the relationships found here are related to the shape of the true variogram (the assumption of local stationarity). These shapes may change if the variogram of soil moisture for larger extents was markedly different.

From a practical hydrologic perspective the case of changing support is probably the most important of the three cases considered here. We will therefore give two examples that illustrate the importance of these scale effects from a modelling perspective. These examples also demonstrate and how the results of this scaling analysis might be used in modelling applications. We will also give an example of the importance of spacing for defining sampling strategies.

\subsection{Modelling}

In hydrologic modelling, the following question is often posed: given variance and correlation length at one scale (support of the measurements) what is the variance and correlation length at another scale (support of the model). Consider the example of a spatially distributed hydrologic model (such as THALES, Grayson et al., 1995) for a small catchment. The size of the model elements is $15 \mathrm{~m}$ and from detailed point samples the true correlation length of soil moisture is known to be $30 \mathrm{~m}$. Question: (a) what is the subgrid variability (i.e. the variability of soil moisture within one model element); (b) what is the variability of the average element soil moisture (i.e. the variability between elements) as simulated by the model assuming it is consistent with the true point scale variability; and (c) what is the correlation length of the patterns as simulated by the model. Solution: For $a_{\text {Supp }} / \lambda_{\text {true }}=0.5$, Fig. 9(a) gives $\sigma_{\text {app,Supp }}^{2}=$ $0.8 * \sigma_{\text {true }}^{2}$. This means that the (normalised) variability within an element is $1-0.8=0.2$ (Eq. A4. (a) The subgrid variability is only $20 \%$ of the total variability and (b) the variability between elements is $80 \%$ of the total variability. (c) Fig. 9(b) suggest that $\lambda_{\text {app }, \text { Supp }}=1.3 * \lambda_{\text {true }}=40 \mathrm{~m}$. In other words, the change of scale considered in this example is not very important in terms of the variance and the correlation length. This sort of analysis would be useful for helping to determine appropriate model element sizes.

Consider a second example of a macroscale model (see, e.g. Sivapalan and Blöschl, 1995) that predicts the soil moisture state in a large region. Specifically, the purpose of the model is to predict the percentage of the land surface that is saturated at a given time. Assume that, at a given time, the true variance of soil moisture is $\sigma_{\text {true }}^{2}=24(\% V / V)^{2}$, mean soil moisture is $38(\% V / V)$ and saturation occurs at a soil moisture threshold of $45(\% V / V)$. Assume also that the true correlation length of soil moisture is $\lambda_{\text {true }}=50 \mathrm{~m}$, and the model element size is $150 \mathrm{~m}$. Question: (a) what is the true percent saturated area and (b) what percent saturated area will the model predict. Solution: (a) For the true soil moisture distribution, the threshold is $(45-38) / \sqrt{24}=1.4$ times the standard deviation above the mean. For a standard normal distribution, the exceedance probability is $P(Z>1.4)=0.08$. This means that $8 \%$ of the land surface is saturated. (b) The model will predict a variance that is smaller than the true variance. For $a_{\text {Supp }} / \lambda_{\text {true }}=3$, Fig. 9(a) gives $\sigma_{\text {app,Supp }}^{2}=$ $0.25 * \sigma_{\text {true }}^{2}=6(\% V / V)^{2}$. For the simulated soil moisture distribution, the threshold is $(45-38) / \sqrt{6}=2.9$ times the standard deviation above the mean. For a standard normal distribution, the exceedance probability is $P(Z>2.9)=0.002$. This means that the model predicts that only $0.2 \%$ of the land surface is saturated. In this example the effect of support is 
Table 3

Measurement scales, as a function of the true correlation length, $\lambda_{\text {true }}$, required for a bias smaller than $10 \%$. Compiled from Figs. 911 as predicted by the relationships given in Appendix A

\begin{tabular}{llll}
\hline Bias $<10 \%$ & Spacing & Extent & Support \\
\hline For $\sigma_{\text {app }}^{2}$ & - & $>6 * \lambda_{\text {true }}$ & $<0.3 * \lambda_{\text {true }}$ \\
For $\lambda_{\text {app }}$ & $<1.5 * \lambda_{\text {true }}$ & $>13 * \lambda_{\text {true }}$ & $<0.3 * \lambda_{\text {true }}$ \\
\hline
\end{tabular}

clearly very important and neglecting this effect can result in gross misinterpretations of data and model results. While these estimates of the saturated area depend on the details of the assumed statistical distribution, the difference in standard deviation suggests that significant scale related differences between estimated saturated area would exist irrespective of the actual statistical distribution. A normal distribution has been assumed above to demonstrate the utility of the scaling relationships. While this is approximately consistent with the observations at Tarrawarra (Western et al., 1998b), further information is required on the shape of the soil moisture probability distribution function and how this varies with catchment wetness, before this approach could be applied generally. It is also important to recognise that this averaging effect can have a significant effect on evapotranspiration, both for the saturated areas and the parts of the landscape that are below saturation. This is because evapotranspiration - soil moisture relationships are typically non-linear. Hence, evapotranspiration calculated from averaged soil moisture may be different from the results of first calculating evapotranspiration at the point scale and then averaging it in a second step. These differences are likely to be closely related to the variance of the underlying soil moisture distribution as well as the ratio of the support and the true correlation length of soil moisture.

\subsection{Sampling}

In hydrologic sampling or measurement a question often posed (at least implicitly) is the following: what spacing, extent and support are needed to insure that the measured soil moisture data will have a minimum bias in terms of their variance and correlation length? If we allow a $10 \%$ bias, Figs. 9-11 give the maximum support, the minimum extent and the maximum spacing as summarised in Table 3. This means that, provided the true correlation length is known a priori, a total of $(13 / 1.5)^{2}=75$ sample points will be needed if the bias is to be lower than $10 \%$. In practical cases the true correlation length will not be known a priori and the number of samples needed may be much larger. In many practical cases it may not be possible to arbitrarily choose the measurement scale due to logistical and other constraints. For these cases, an important question that is closely related to the above one may be: given the apparent variance and correlation length from the data what is the true underlying variance and correlation length? Consider the example of a $7 \mathrm{~km}^{2}$ catchment in which 69 point samples have been taken. From these samples, a practical range of $300 \mathrm{~m}$ has been estimated which is consistent with an apparent correlation length of about $100 \mathrm{~m}$. Question: (a) is this estimate biased and (b) if so, how many samples would be needed for an unbiased estimate. Solution: The solution is found by trial and error from Fig. 11. Eq. (3) gives $a_{\text {Spac }}=320 \mathrm{~m}$. If we assume that the true correlation length is $65 \mathrm{~m}, a_{\text {Spac }} / \lambda_{\text {true }}=4.9$, Fig. 11 gives $\lambda_{\text {app }, \text { Spac }} / \lambda_{\text {true }}=1.55$ when using the results for survey S3 (23 Feb 96) which is a solution to the above problem. This means that the data should have been collected at a scale of $1.5 \times 65 \mathrm{~m}=100 \mathrm{~m}$ (Table 3 ) to minimise the bias caused by the spacing. Therefore, a total of 700 samples will be necessary for the $7 \mathrm{~km}^{2}$ catchment of the example assumed here. It is interesting to note that the predictive relationship in Fig. 11 (Eq. A16 does not give a solution to the example assumed here. In essence this is related to the fact that it is difficult to infer information at scales much smaller than those of the actual data.

When designing a field experiment to measure the spatial variance and correlation length, it is necessary to determine the spacing, support and extent at which the measurements will be made. Ideally the support should be small, the spacing small and the extent large, compared with the true correlation length. In practice the support is usually determined by the measurement technique and is chosen by default when the measurement technique is chosen. Usually in field studies, the support is much smaller than the correlation length, with the exception being cases where the catchment is used as an instrument (i.e. in runoff studies). 
This leaves the spacing and extent to be determined. The possible combinations of spacing and extent are likely to be limited by the resources available. However, there are some important limits. The spacing should not exceed $1.5 \times \lambda_{\text {true }}$. If a spacing greater than about $1.5 \times \lambda_{\text {true }}$ is used there will be insufficient resolution in the data to define the true correlation length (it is not possible to obtain $\lambda_{\text {true }}$ from $\lambda_{\text {app }, s p a c}$ using the predictive relationship in Fig. 11 if the spacing is too large). It should be noted that a finer spacing might be required if the aim is to resolve organised features, such as connectivity, in the spatial field. Fig. 10 indicates that the extent needs to be quite large compared to the correlation length (at least $13 \times$ $\lambda_{\text {true }}$ for a bias less than 10\%). To apply the predictive relationships in Figs. 9-11 to the problem of sampling design an estimate of $\lambda_{\text {true }}$ is required. This may be obtained from previous studies, a pilot study or by careful consideration of surrogate information on the features likely to control the spatial field of interest. For example, the topography might be relevant for obtaining an initial estimate of $\lambda_{\text {true }}$ for soil moisture. It is also important to sample sufficient points to ensure that the variogram can be estimated accurately (Western et al., 1998b).

The resampling analysis and the examples considered here have been simplified in that a change in scale of a single component of the scale triplet (either spacing, extent, or support) has been considered. In some real world applications, a combined change of scale will be called for when going from the scale of the data (measurement scale) to the scale of the predictions (modelling scale). A combined change of scale (either upscaling or downscaling) of more than one component of the scale triplet will have an effect that is a mix of the individual effects. For example, if we increase both extent and spacing, it is clear that the combined effect will be an increase in the variance and an increase in the apparent correlation length. In fact upscaling any combination of spacing, extent, or support will increase the correlation length. However, the effect on the variance of upscaling both extent and support will depend on the relative importance of the two individual effects.

The effect of scaling on the variance and the correlation length has been analysed here for the case of a small catchment. The main advantage of this study is that real high resolution field data have been used and it has been shown that geostatistical techniques are indeed applicable for real soil moisture fields, even if they are spatially organised rather than random. It is clear that the principles shown here will also be applicable at much larger scales. However, for these scales it will be far more difficult to obtain high quality spatial soil moisture data. The principles shown here will also be applicable to a much wider range of scales than the two orders of magnitude examined in this study. However, for a much wider range of scales, the assumption of a stationary exponential variogram will have to be reconsidered. For example, if the scales are increased beyond that of the Tarrawarra catchment, new sources of variability will come in such as differences in land use, vegetation, soil type and geology. For a range of, say, four orders of magnitude a nested exponential variogram may be more appropriate than a single exponential variogram. Work geared towards determining the larger scale variability in the Tarrawarra region is under way (Western et al., 1997).

\section{Conclusions}

In this paper the effect on the apparent spatial statistical properties of soil moisture (variance and correlation length) of changes in the measurement scale (in terms of spacing, extent and support) has been examined using a resampling analysis. 'Spacing' refers to the distance between samples; 'extent' refers to the overall coverage; and 'support' refers to the integration area. For the ideal case of very small spacings, very large extents and very small supports, the apparent variance and the apparent correlation length are close to their true values. However, as the spacing increases, the extent decreases or the support increases, bias is introduced. Apparent correlation lengths always increase when increasing spacing, extent or support. Bias in the correlation length is significant once spacing exceeds about twice the true correlation length, once extent is smaller than about 5 times the true correlation length, or once support exceeds about $20 \%$ of the true correlation length. The effect of extent on the correlation length is the most important one of the three. The apparent variance increases with increasing extent, decreases with increasing support, and does not change with 
spacing. Bias in the variance is significant once extent is smaller than about 5 times the true correlation length, or once support exceeds about $20 \%$ of the true correlation length. The effect of support on the variances is the more important one of the two. All these sources of bias are a function of the ratio of the measurement scale (in terms of spacing, extent and support) and the scale of the natural variability (i.e. the true correlation length of soil moisture or process scale). The effect of the modelling scale (in terms of spacing, extent and support) is similar to that of the measurement scale.

The results of the resampling analysis have been compared to predictions of bias estimated using standard geostatistical techniques of regularisation and variogram analysis. Specifically, we examined whether these techniques are applicable to soil moisture patterns. This was done because soil moisture patterns exhibit spatial organisation, while geostatistical techniques are based on the assumption of soil moisture being a random variable. The comparison indicates that these techniques are indeed applicable for organised soil moisture patterns. The predictive performance of the relationships given in Appendix $\mathrm{A}$ is as good for surveys where soil moisture shows spatial organisation as for surveys where the soil moisture patterns are spatially random. This comparison lends some credence to these geostatistical techniques for hydrologic applications. A number of examples are given to illustrate the potential application and the importance of the spatial scaling of soil moisture. These examples also illustrate how geostatistical methods can be used in both modelling and sampling design to assess the bias introduced by the measurement scale and the modelling scale in terms of spacing, extent and support.

\section{Acknowledgements}

The Tarrawarra catchment is owned by the Cistercian Monks (Tarrawarra) who have provided free access to their land and willing cooperation throughout the project. Funding for the above work was provided by the Australian Research Council (project A39531077), the Cooperative Research Centre for Catchment Hydrology, the Oesterreichische Nationalbank, Vienna (project 5309) and the Australian
Department of Industry, Science and Tourism, International Science and Technology Program.

\section{Appendix A}

The analysis in the paper consists of three main steps. The first step is an analysis of the full data set, which gives the "true" variogram. The second step is a resampling analysis in which the empirical values of the apparent variance and integral scale are estimated. The third step consists of estimating the theoretical values of the apparent variance and integral scale directly from the "true", variogram. This appendix gives the methods used in the third step.

We use an exponential variogram without nugget for representing the "true", spatial variability of soil moisture. The reason for doing this is that, in practice, there are often insufficient data for inferring accurate nugget values and hence, often nugget is assumed to be zero. For consistency, we also use zero nuggets when fitting variograms in the resampling analysis and for the direct estimation of the theoretical variance and integral scale from the "true" variogram described in this appendix. The exception is the extent case as nugget can be important for small extents. In the extent case we therefore also examine a variogram with a non-zero nugget. A second assumption is that of local stationarity, i.e. a sill of the apparent variogram exists at all scales and is equal to the (apparent) variance of the data at that scale.

The apparent variance, $\boldsymbol{\sigma}_{a p p}^{2}$, and the apparent integral scale, $\boldsymbol{I}_{a p p}$, for each of the cases of the scale triplet "support, extent, and spacing" are calculated as follows. For clarity, the subscripts of the scales $\boldsymbol{a}_{\text {Supp }}, \boldsymbol{a}_{\text {Ext }}$, and $\boldsymbol{a}_{\text {Spac }}$ are not written as they are obvious from the section headings.

\section{A.1. True variogram (no nugget)}

The true variogram, $\gamma_{\text {true }}$, is assumed to be of the form:

$\gamma_{\text {true }}=\sigma_{\text {true }}^{2} \cdot\left(1-\exp \left(\frac{-h}{\lambda_{\text {true }}}\right)\right)$

where $h$ is the lag. The sill is set to the variance of the full data set, $\sigma_{\text {true }}^{2}$, and the correlation length, $\lambda_{\text {true }}$, is estimated by fitting A.1 to the empirical variogram of 
the full data set. The true integral scale

$I_{\text {true }}=\int_{0}^{\infty} 1-\frac{\gamma_{\text {true }}(x)}{\sigma_{\text {true }}^{2}} \mathrm{~d} x$

is simply

$I_{\text {true }}=\lambda_{\text {true }}$

\section{A.2. Support (regularisation)}

The idea of regularisation is to obtain the properties of an averaged process by running a linear filter over the original point process, i.e. simple arithmetic averaging applies. The filter is represented by a square of side length $a$ which is the support. The variance of the averaged process, $\sigma_{a p p, \text { Supp }}^{2}$, is the total variance minus the variance within the square, $\sigma_{\text {within }}^{2}$, (Journel and Huijbregts, 1978):

$\sigma_{\text {app }, \text { Supp }}^{2}=\sigma_{\text {true }}^{2}-\sigma_{\text {within }}^{2}$

For an isotropic process $\sigma_{\text {within }}^{2}$ can be obtained as (Rodríguez-Iturbe and Mejía, 1974):

$\sigma_{\text {within }}^{2}=\int_{0}^{R \max } \gamma_{\text {true }}(r) \cdot f_{1}(r \mid a) \mathrm{d} r$

where $r$ is the distance between two randomly chosen points in the square, $R_{\max }$ is the maximum distance in the square, and $f_{1}(r \mid a)$ is the probability density function (pdf) of the distances in the square of side length $a$. $f_{1}$ has been derived by Ghosh (1951) as

$f_{1}(r)=\frac{4 r}{a^{4}} \phi_{1}(r)$

where

$\phi_{1}(r)=\frac{1}{2} \pi a^{2}-2 a r+\frac{1}{2} r^{2}$

for $0 \leq r \leq a$

$$
\begin{aligned}
\phi_{1}(r)= & a^{2}\left\{\sin ^{-1} \frac{a}{r}-\cos ^{-1} \frac{a}{r}\right\}+2 a \sqrt{r^{2}-a^{2}} \\
& -\frac{r^{2}+2 a^{2}}{2}
\end{aligned}
$$

for $a<r \leq \sqrt{2} a$

The variogram of the averaged process can be found in an analogous fashion to Eq. A5 by

$\gamma_{\text {app }, \text { Supp }}(h, a)=\int_{0}^{\text {Rmax }} \gamma_{\text {true }}(r) \cdot f_{2}(r \mid h, a) \mathrm{d} r-\sigma_{\text {within }}^{2}$

where $f_{2}$ is the pdf of distances of two points each of which is randomly located in one of two squares where the two squares are separated by a (centre-tocentre) distance $h$ and have side lengths $a . f_{2}$ was evaluated by Sivapalan (1986) as:

$f_{2}(r)=\frac{2 r}{a^{4}} \phi_{2}(r)$

where

$\phi_{2}(r)=a \sqrt{r^{2}-e^{2}}-\frac{(r-e)^{2}}{2}-e a \cos ^{-1}\left(\frac{e}{r}\right)$

for $e \leq r \leq \sqrt{a^{2}+e^{2}}$

$\phi_{2}(r)=\frac{a^{2}}{2}+e\left(r-\sqrt{r^{2}-a^{2}}\right)-e a \sin ^{-1}\left(\frac{a}{r}\right)$

for $\sqrt{a^{2}+e^{2}} \leq r \leq h$

$\phi_{2}(r)=a g \cos ^{-1}\left(\frac{h}{r}\right)-e a\left[\sin ^{-1}\left(\frac{a}{r}\right)-\cos ^{-1}\left(\frac{h}{r}\right)\right]$

$-e \sqrt{r^{2}-a^{2}}-2 a \sqrt{r^{2}-h^{2}}+\frac{a^{2}}{2}+h^{2}+r^{2}-g r$

for $h \leq r \leq \sqrt{a^{2}+h^{2}}$

$\phi_{2}(r)=a g \sin ^{-1}\left(\frac{a}{r}\right)-a^{2}+g \sqrt{r^{2}-a^{2}}-g r$

for

$\sqrt{a^{2}+h^{2}} \leq r \leq g$

$\phi_{2}(r)=a g\left[\sin ^{-1}\left(\frac{a}{r}\right)-\cos ^{-1}\left(\frac{g}{r}\right)\right]+g \sqrt{r^{2}-a^{2}}$

$+a \sqrt{r^{2}-g^{2}}-\frac{a^{2}+r^{2}+g^{2}}{2}$

for $g \leq r \leq \sqrt{a^{2}+g^{2}}$ with $e=h-a$ and $g=h+a$

The variogram of the averaged process can now be used to calculate numerically the integral scale of the 
averaged process:

$I_{\text {app }, \text { Supp }}=\int_{0}^{\infty} 1-\frac{\gamma_{a p p, \text { Supp }}(x)}{\sigma_{\text {app }, \text { Supp }}^{2}} \mathrm{~d} x$

\section{A.3. Extent}

The apparent variance of a process with the extent limited to a square of side length $a$ is the variance within the square (see Eq. A4 and Eq. A5):

$$
\sigma_{a p p, E x t}^{2}=\sigma_{\text {within }}^{2}
$$

The apparent variogram is identical with the true variogram for lags that are small as compared to the extent, $a$, and it is equal to the apparent variance for lags that are large as compared to the extent. This is consistent with the exact solution. For intermediate lags it is assumed here, for simplicity, that there is a sharp crossover at $L$ where $\gamma_{\text {true }}(L)=\sigma_{a p p, E x t}^{2}$

$$
\begin{aligned}
& \gamma_{\text {app }, E x t}=\gamma_{\text {true }} \\
& \text { for } h \leq L \\
& \gamma_{\text {app }, E x t}=\sigma_{a p p, E x t}^{2} \\
& \text { for } h>L
\end{aligned}
$$

where

$L=-\lambda_{\text {true }} \cdot \ln \left(1-\frac{\sigma_{\text {app }, E x t}^{2}}{\sigma_{\text {true }}^{2}}\right)$

The integral scale is:

$I_{a p p, E x t}=\int_{0}^{\infty} 1-\frac{\gamma_{a p p, E x t}(x)}{\sigma_{a p p, E x t}^{2}} \mathrm{~d} x$

which can be evaluated as:

$$
\begin{gathered}
I_{a p p, E x t}=\frac{\lambda_{\text {true }}}{\sigma_{\text {app }, \text { Ext }}^{2}} \cdot\left[\sigma_{\text {app }, E x t}^{2}+\left(\sigma_{\text {true }}^{2}-\sigma_{\text {app }, \text { Ext }}^{2}\right)\right. \\
\left.\cdot \ln \left(1-\frac{\sigma_{\text {app }, \text { Supp }}^{2}}{\sigma_{\text {true }}^{2}}\right)\right]
\end{gathered}
$$

\section{A.4. Spacing}

For any correlation structure, the apparent variance is equal to the true variance:

$\sigma_{\text {app }, \text { Spac }}^{2}=\sigma_{\text {true }}^{2}$

This is because $\sigma_{a p p, S p a c}^{2}$ can be interpreted as the variance of $n$ point samples which is close to the population variance provided $n$ is not too small (Matalas, 1967). Here, $n$ is large because the case for changing the spacing is, notionally, for large extents. The apparent variogram is estimated based on the following simple assumptions: For lags larger than the spacing, $a$, the apparent variogram is assumed to be identical with the true variogram. For lags smaller than the spacing, $a$, the variogram is assumed to be linear between the origin $\gamma(0)=0$ and $\gamma(a)$. This assumption is made because, in many practical cases, when estimating the empirical variogram there are only a small number of pairs of points for lags smaller than the spacing (Russo and Jury, 1987) and a straight line is the simplest approximation.

$\gamma_{a p p, S p a c}(h)=\frac{h}{a} \gamma_{\text {true }}(a)$

for $h \leq a$

$\gamma_{\text {app }, \text { Spac }}(h)=\gamma_{\text {true }}(h)$

for $h>a$

From Eq. A15 the integral scale can be calculated as:

$I_{\text {app }, \text { Spac }}$

$$
=\lambda_{\text {true }}\left[\exp \left(\frac{-a}{\lambda_{\text {true }}}\right)+\frac{a}{2 \lambda_{\text {true }}} \cdot\left(1+\exp \left(\frac{-a}{\lambda_{\text {true }}}\right)\right)\right]
$$

For small spacings (as compared to the correlation length), $I_{a p p, S p a c}$ is controlled by the correlation length, $\lambda_{\text {true }}$, while for large spacings, $I_{a p p, S p a c}$ is controlled by the spacing, $a$.

\section{A.5. Extent (with nugget)}

For the case of changing the extent, and for small extents (as compared to the correlation length), it may be necessary to correct for the effect of nugget. The true variogram (Eq. A1) now becomes:

$$
\gamma_{\text {true }, \text { nugg }}=\sigma_{\text {nug }}^{2}+\left(\sigma_{\text {true }}^{2}-\sigma_{\text {nug }}^{2}\right) \cdot\left(1-\exp \left(\frac{-h}{\lambda_{\text {true }}}\right)\right)
$$

where $\sigma_{n u g}^{2}$ is the nugget and $\lambda_{\text {true }}$ is the correlation 
length estimated by fitting A.1 to the empirical variogram of the full data set. In other words, the effect of the nugget on $\lambda_{\text {true }}$ is neglected and hence the same value of $\lambda_{\text {true }}$ is used in Eqs. A.1 and A.17. The true integral scale is

$I_{\text {true }}=\lambda_{\text {true }}\left(1-\frac{\sigma_{\text {nug }}^{2}}{\sigma_{\text {true }}^{2}}\right)$

The effect of the nugget can now be accommodated by modifying Eq.. A10 which gives the apparent variance:

$\sigma_{\text {app }, E x t, n u g}^{2}=\sigma_{\text {app }, \text { Ext }}^{2}\left(1-\frac{\sigma_{\text {nug }}^{2}}{\sigma_{\text {true }}^{2}}\right)+\sigma_{\text {nug }}^{2}$

and by modifying Eq. A13 which gives the apparent integral scale:

$$
\begin{aligned}
& I_{\text {app }, \text { Ext }, \text { nug }}=\frac{I_{\text {true }}}{\sigma_{\text {app }, \text { Ext }, \text { nug }}^{2}}\left(\frac{\sigma_{\text {true }}^{2}}{\sigma_{\text {true }}^{2}-\sigma_{\text {nug }}^{2}}\right) \\
& \times\left[\sigma_{\text {app }, \text { Ext }, \text { nug }}^{2}-\sigma_{\text {nug }}^{2}+\left(\sigma_{\text {true }}^{2}-\sigma_{\text {app }, \text { Ext,nug }}^{2}\right)\right. \\
& \left.\cdot \ln \left(\frac{\sigma_{\text {true }}^{2}-\sigma_{\text {app }, \text { Ext,nug }}^{2}}{\sigma_{\text {true }}^{2}-\sigma_{\text {nug }}^{2}}\right)\right]
\end{aligned}
$$

\section{References}

Beckie, R., 1996. Measurement scale, network sampling scale and groundwater model parameters. Water. Resour. Res. 32, 65-76.

Blöschl, G., 1998. Scale and Scaling in Hydrology — a framework for thinking and analysis. John Wiley, Chichester, in press.

Blöschl, G., Sivapalan, M., 1995. Scale issues in hydrological modelling - a review. Hydrol. Processes 9, 251-290.

De Troch, F.P., Troch, P.A., Su, Z., Lin, D.S., 1996. Application of remote sensing for hydrological modelling. In: Abbott, M.B., Refsgaard, J.C. (Eds.), Distributed Hydrological Modelling. Kluwer Academic Press, pp. 165-192.

Dunne, T., Moore, T.R., Taylor, C.H., 1975. Recognition and prediction of runoff-producing zones in humid regions. Hydrol. Sci. Bull. 20, 305-327.

Feder, J., 1988. Fractals. Plenum, New York and London, 283 pp.

Gelhar, L.W., 1986. Stochastic subsurface hydrology from theory to applications. Water Resour. Res 22, 135S-145S.

Gelhar, L.W., 1993. Stochastic Subsurface Hydrology. Prentice Hall, Englewood Cliffs, NJ, 390 pp.

Georgakakos, K.P., 1996. Soil moisture theories and observations, J. Hydrol. 184 (special issue).
Ghosh, B., 1951. Random distances within a rectangle and between two rectangles. Bull. Calcutta Math. Soc. 43, 17-24.

Grayson, R.B., Blöschl, G., Moore, I.D., 1995. Distributed parameter hydrologic modelling using vector elevation data: THALES and TAPES-C. Chapter 19. In: V. P. Singh (Editor), Computer Models of Watershed Hydrology. Water Resources Publications, Highlands Ranch, Colorado, pp. 669-696.

Isaaks, E.H., Srivastava, R.M., 1989. An Introduction to Applied Geostatistics. Oxford University Press, New York, Oxford, 561 pp.

Jenkins, G.M., Watts, D.G., 1968. Spectral analysis and its applications. Holden-Day, San Francisco, 525 pp.

Journel, A.G., Huijbregts, C.J., 1978. Mining Geostatistics, Academic Press, London, 600 pp.

Klemeš, V., 1974. The Hurst phenomenon: a puzzle?. Water Resour. Res. 10, 675-688.

Matalas, N.C., 1967. Mathematical Assessment of Synthetic Hydrology. Water Resour. Res. 3 (4), 937-945.

Rodríguez-Iturbe, I., Mejía, J.M., 1974. On the transformation from point rainfall to areal rainfall. Water Resour. Res. 10, 729-735.

Rodríguez-Iturbe, I., Vogel, G.K., Rigon, R., Entekhabi, D., Castelli, F., Rinaldo, A., 1995. On the spatial organization of soil moisture fields. Geophys. Res. Lett. 22, 2757-2760.

Russo, D., Jury, W.A., 1987. A theoretical study of the estimation of the correlation scale in spatially variable fields. 1. Stationary fields. Water Resour. Res. 23, 1257-1268.

Schmugge, T.J., Jackson, T.J., 1996. Soil moisture variability. In: Stewart, J.B., et al. (Eds.), Scaling up in Hydrology using Remote Sensing. Wiley, Chichester, pp. 183-192.

Sivapalan, M., 1986. Scale Problems in Rainfall, Infiltration and Runoff Production. PhD dissertation, Dept. of Civil Engng, Princeton University, $271 \mathrm{pp}$.

Sivapalan, M., Blöschl, G., 1995. An overview of large scale hydrological modelling. MODSIM 95, International Congress on Modelling and Simulation, Proceedings Vol. 1. Modelling and Simulation Society of Australia, Canberra, pp. 139-149.

Stewart, J.B., Engman, E.T., Feddes, R.A., Kerr, Y. (Eds.), 1996. Scaling up in Hydrology using Remote Sensing. Wiley, Chichester, 255 pp.

Vanmarcke, E., 1983. Random Fields: Analysis and Synthesis. The MIT Press, Cambridge, Massachusetts, 382 pp.

Western, A.W., Grayson, R.B., 1998. The Tarrawarra data set: soil moisture patterns, soil characteristics and hydrological flux measurements. Water Resour. Res., in press.

Western, A.W., Grayson, R.B., Blöschl, G., Willgoose, G.R., McMahon, T.A., 1998a. Observed spatial organisation of soil moisture and relation to terrain indices. Water Resour. Res., in press.

Western, A.W., Blöschl, G., Grayson, R.B., 1998b. Geostatistical characterisation of soil moisture patterns in the Tarrawarra catchment. Journal of Hydrology 205, 20-37.

Western, A.W., Grayson, R.B., Blöschl, G., Willgoose, G.R., McMahon, T.A., 1997. The Tarrawarra project: high resolution spatial measurement and analysis of hydrological response. In: MODSIM 97, International Congress on Modelling and Simulation held at Hobart, Tasmania. Modelling and Simulation Society of Australia, Canberra. 